\title{
American Indian Male College Students Perception and Knowledge of Human Papillomavirus (HPV)
}

Felicia Schanche Hodge*

UCLA School of Nursing, Los Angeles, CA 90095-1702, USA

"Corresponding author: Felicia Schanche Hodge, Professor, UCLA School of Nursing, Los Angeles, CA 90095-1702, USA, Tel: (310) 267-2255; E-mail: fhodge@sonnet.ucla.edu

Rec date: 08 Feb 2014; Acc date: 21 Feb 2014; Pub date: 25 Feb 2014

Copyright: (C) 2014 Hodge FS. This is an open-access article distributed under the terms of the Creative Commons Attribution License, which permits unrestricted use, distribution, and reproduction in any medium, provided the original author and source are credited.

\begin{abstract}
The authors explored American Indian male college students' HPV attitudes, perceptions and knowledge of HPV prevention and transmission. Eight focus groups with male and female students ages 19-26 were conducted at four Southwest universities. Audio recordings were transcribed and analyzed using Grounded Theory methods. American Indian males reported little or no responsibility for HPV prevention and transmission $(p=0.048)$ and held poor personal risk perception $(p=0.0001)$. Male students also had low levels of HPV knowledge $(p=0.003)$ and lenient attitudes, indicating an overall disregard for safer sexual practices. Misinformation regarding HPV vaccination played a significant part in their lack of intention to modify sexual behaviors. Cultural barriers to obtaining HPV information and sources of HPV education were identified, as was preference for receiving HPV information. We recommend school-based culturally sensitive HPV prevention programs that focus on improving responsible safer sexual practices by educating students in activities to reduce cancer and STI disease burden. Emphasis on preventing genital warts, other STIs, as well as HPV-related cancers found in males and females may increase acceptability and responsibility of HPV prevention measures among male American Indian students.
\end{abstract}

Keywords: American indians; Males; College students; Human papillomavirus; HPV; Knowledge; Vaccination; Risk perceptions; Responsibility; Prevention

\section{Introduction}

Human papilloma virus (HPV) is a sexually transmitted infection. It is known to cause almost all cervical cancers and genital warts. Quadrivalent HPV vaccine became available for prophylaxis of young women in 2006, only recently were recommendations issued for prophylasis of young men. HPV vaccination intended for male youth aged $11-21$ years [1] has focused attention to the need for better acceptability for HPV vaccination and ways to improve compliance among high risk males, especially those in hard to reach populations. More effective delivery of HPV vaccinations to targeted young male populations requires assessment of barriers to care, which entails understanding the population's attitudes, understanding their perception of risk, assessing their knowledge of HPV prevention and transmission to meet the needs of the target population, in this case young American Indian men. HPV prevention strategies include abstinence, barrier protection, limiting sexual partners and, most recently, vaccination. Gardasil, the first HPV vaccine, was developed to prevent cervical cancer, precancerous genital lesions, and genital warts caused by certain strains of the virus [2]. The vaccine covers the HPV variants that cause approximately 75 percent of all cervical cancers and 90 percent of genital warts [3] The HPV vaccine was initially licensed for and recommended for routine prophylactic use in young women in the age range, 12 to 26 years, with administration in a series of three injections prior to sexual initiation [4]. Males were not included in the vaccine's original recommendation, even though vaccinating males can potentially prevent cervical cancer in their intimate partners. Newer vaccines, such as Cervarix, target both genders in vaccine regimens. Gardasil has revised its protocol; it is now approved by the Food and Drug Administration (FDA) as prophylaxis against genital warts in boys and young men aged 9 to 26 years [4].

American Indians suffer from some of the highest rates of sexuallytransmitted infections (STI) in the nation. Rates of chlamydia and gonorrhea are more than 4 times higher than for the U.S. white population [5], and the case rate of C. trachomatis is 6 times the overall U.S. rate [6]. HPV prevalence is also higher among American Indian and Alaska Native women than among Hispanic women. Similar prevalence of HPV high-risk strains 16 and 18 is seen in American Indian and Alaska Natives, as compared with Hispanic and African American women [7]. It has been demonstrated that HPV DNA can be detected in approximately $99 \%$ of all invasive cervical cancers [8], and unfortunately, the cervical cancer mortality rate among American Indian and Alaska Natives is reported to be 2 to 5 times that of the general population and is a leading cause of cancer mortality in this population [9].

It is estimated that nearly 50 percent of all sexually active individuals will become infected with HPV within their lifetime [10]. While HPV-related sequelae primarily impact women's health, men are also at risk of contracting HPV infection through unprotected sexual contact. Although HPV infection is often without visible evidence, men's HPV infection plays an important role in the transmission to females. HPV is a known risk factor for and the leading cause of cervical cancer in women, it has also been shown to cause cancer in males, such as cancers of the penis and anus, as well as oropharyngeal cancer $[11,12]$. Although presently somewhat rare, HPV-related oropharyngeal cancers among men are expected to surpass the number of cervical cancer rates among women by the year $2020[13]$. 
HPV infection is typically asymptomatic, and research studies have reported that both males and females generally report no or limited awareness of HPV infection and its association to several serious cancers [14-16]. Low knowledge levels about HPV and STI may contribute to low perceived risk, as well as non-participation in HPV education and vaccination programs. However, with the recent availability of HPV vaccination, the serious cancer-related consequences to HPV can now be considered a preventable disorder.

Increased rates of cervical cancer among American Indian women, and reports that they are more likely to contract and die from cervical cancer than non-Hispanic white women [17] support the need for improving HPV knowledge and awareness in both genders of American Indian populations. Targeted education, prevention and control efforts can lead to safer sexual behaviors and higher acceptance of HPV vaccines. Improving HPV knowledge and awareness of both males and females can lead to enormous health benefits thereby decreasing morbidity and mortality associated with HPV transmission between heterosexual partners, especially related to cervical cancer and the occurrence of genital warts, as well as other STIs. Savings realized in the treatments for genital warts, pre-invasive cervical lesions, and cervical cancer would contribute to reduced health care expenditures [17].

In this article, we report an investigation of American Indian male college students' HPV attitudes, perceptions and knowledge of HPV prevention and transmission. The study was conducted among American Indian students attending colleges/universities in California and Arizona. This American Indian population was selected because they are age eligible for HPV vaccination and for the high availability and concentration of American Indian students who have matriculated to West and Southwest colleges. This paper reports on a preliminary exploration of HPV among an important and often overlooked at risk subgroup, American Indian males, and the unique issues and barriers they experience, as compared to female participants.

\section{Materials and Methods}

\section{Study population and design}

American Indian college students were recruited from four large California and Arizona colleges/universities (Arizona State University, University of Arizona, University of California at Berkeley, and the University of California, Los Angeles) in 2009 to participate in a study about HPV infection. Fifty-three American Indian college students were recruited. Twenty-one were males and 32 were females. Both male and female students were recruited to attend focus groups by word of mouth and through the use of flyers posted at university sites (including American Indian Studies departments, student unions, and news boards). Inclusion criteria included age ( $>18$ years), enrolled college student status, and self-identification as American Indian. Exclusion criteria are non-students, youth under the age of 18, and non-Indians. Participants read and signed a consent form and they received a verbal and written overview of the project, told of their voluntary participation, and assured that participation would not influence academic grading, course credit, or other university services. Each participant received a gift card valued at $\$ 25.00$ at the end of the focus group session. Institutional Review Board approvals were obtained from the University of California, Los Angeles before commencing the study.
Table 1 reports the socio-demographic characteristics of the sample. Males ranged in age from 19 to 26 years, they were slightly younger than the female participants. The largest proportion of the sample was recruited from the University of Arizona (8 males, 13 females), followed by Arizona State University (10 males, 6 females), University of California at Berkeley ( 3 males, 8 females) and the University of California, Los Angeles (0 males, 5 females). The majority of male participants were affiliated with a Southwest tribe (85.7\%), and other males represented tribes from California, Oklahoma and the Northern Plains areas.

\begin{tabular}{|l|l|l|l|}
\hline Characteristics & $\begin{array}{l}\text { Male } \\
\mathrm{n}=23(40.4)\end{array}$ & $\begin{array}{l}\text { Female } \\
\mathrm{n}=34(59.6)\end{array}$ & $\mathrm{p}$-value \\
\hline Demographics & Mean (SE) & Mean (SE) & \\
\hline Age & $\begin{array}{l}21.28 \\
(12.63)\end{array}$ & $\begin{array}{l}26.91 \\
(16.93)\end{array}$ & \\
\hline & $\mathrm{n} \%$ & $\mathrm{n} \%$ & \\
\hline Tribal Affiliation: & $19(82.6)$ & $21(61.8)$ & \\
Arizona tribe & $0 .(00.0)$ & $5(14.7)$ & \\
$\begin{array}{l}\text { California tribe } \\
\text { Other tribe }\end{array}$ & $4(17.4)$ & $8(23.5)$ & \\
\hline
\end{tabular}

Knowledge: (higher numbers indicate higher knowledge)

\begin{tabular}{|l|l|l|l|}
\hline Method of transmission & $1(4.4)$ & $12(35.3)$ & $0.003^{*}$ \\
Vaccine eligibility & & & \\
Vaccine is series of shots & & & \\
HPV easy to get & & & \\
HPV is easily treated & & & \\
HPV is serious among Als & & & \\
\hline
\end{tabular}

Perception of Risk: (higher numbers indicate lower perception of risk)

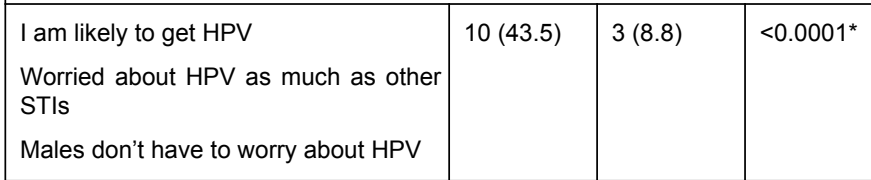

Prevention: (higher numbers indicate stronger prevention beliefs/activities)

\begin{tabular}{|l|l|l|l|}
\hline I have received the vaccine & $14(60.9)$ & $26(67.7)$ & $0.048^{*}$
\end{tabular}

I plan on getting the vaccine

Would recommend vaccine to a friend

I do what I can to prevent myself from getting HPV

Table 1: Characteristics of American Indian College Student Knowledge and Attitude of HPV Prevention and Transmission by Gender $(\mathrm{N}=57)$

*: significant difference between classifiers based on Chi-square or Fisher's Exact test

at alpha $=0.05$ 


\section{Description of focus groups}

Focus groups were designed following an extensive review of the literature, which guided prompts and probing questions for discussion of HPV. Constructs of interest for use in the focus groups included: (a) knowledge, (b) preventive behaviors (safer sexual behaviors, HPV vaccines), (c) perceived risk, (d) barriers, and (e) preferred sources of information. All focus groups were guided by these constructs and questions were asked that probed within these areas of focus.

Two trained female American Indian graduate student facilitators coordinated and conducted the mixed gender focus groups. Each focus group was one hour and a half in duration and was audio-recorded, then transcribed verbatim for data collection and analysis purposes. Focus groups took place on college campuses within community rooms in the Student Union or in conference rooms at the American Indian Studies department. Transcriptions for all male and female participants' responses and narrative contributions were identified and analyzed independently by three project researchers. To identify recurring themes and patterns within the data, dialogue was coded line-by-line using constant comparative coding techniques following content analysis procedures [18]. Themes were then formed into categories capturing key words, thoughts, attitudes, and recommendations; then, possible relationships between categories were analyzed. Conceptual saturation was considered to be accomplished when no new codes were generated. The researchers completed separate analyses of the data independently and then jointly re-confirmed the themes and concepts. No major discrepancies were identified in the themes.

\section{Results}

\section{Demographics}

Fifty-three American Indian college students, 21 (39.6\%) of whom were males and $32(60.4 \%)$ were females, participated in eight focus groups. Table 1 reports the socio-demographic characteristics of the sample. Males ranged in age from 19 to 26 years, they were slightly younger than the female participants. The largest proportion of the sample was recruited from the University of Arizona (8 males, 13 females), followed by Arizona State University (10 males, 6 females), University of California at Berkeley (3 males, 8 females) and the University of California, Los Angeles (0 males, 5 females). The majority of male participants were affiliated with a Southwest tribe $(85.7 \%)$, and other males represented tribes from California, Oklahoma and the Northern Plains areas.

\section{Knowledge}

Only a few of the male students reported that they had any knowledge of HPV and few were aware of the HPV vaccine. No participant could provide a definition of HPV infection. One male student stated, "I never heard of it until now." Others stated that they had not seen any television commercials or media information about the HPV vaccination. They stated they were not aware of anyone who had obtained the HPV vaccine. Many of the participants had misconceptions about the virus ("I think it can turn into some herpes form or there's bumps."). The male participants were aware of their lack of knowledge, resulting in requests for available information on HPV ("Just tell me everything").

\section{Perceived risk}

Unlike their female counterparts, male participants believed that they were not at risk for HPV infection - rather they expressed denial about how HPV is transmitted. One participant wondered if "(men) might not be affected" by the virus, because "I know that it is more common among women." Denying HPV transmission with heterosexual intimacy, another reasoned, "It's never like, we (males) need to prevent this..." He could not conceive that men might contribute to HPV infection in women, rather his remark suggested it was an impossibility among males. Other males confirmed similar misconceptions, "I didn't take it seriously because I don't think it really happens in males."

\section{Perceived responsibility}

Acknowledging a role in or accepting any level of responsibility for the prevention and control of HPV for themselves and their female sexual partners was found to be low among the male participants. Most reported not being attentive to HPV education; they considered HPV prevention more important for women, and therefore not within their role as men. "I guess (HPV programs are designed) in particular for women because it seems they are the ones that are affected the most by this." Some American Indian males in the study conveyed lack interest or participation in safer sexual practices, such as barrier methods or abstinence, "(Like) we're going to get pregnant?" Some reported advising others that protection was important ("Yes, I talked to my little brothers about (STDs)...I'm the oldest with my siblings, so I want to make sure all my brothers are aware of it... and surprisingly, at ages 18 and $19 .$. They had no idea about this stuff...they had no idea about what they were and how they get them and stuff. So when I talked to them about it, they were kind of like whoa, hey maybe I should slow down. So as an older brother, yes I do believe I should talk to them about my brothers about it....Make them aware about STDs and stuff like that.")

When the group discussions focused on the HPV vaccination, which was targeted only to young girls and women at that time, a male participant explained his lack of awareness regarding the issue, "Even after sixth grade, how many of us actually paid attention to that?" When asked about the possibility of HPV prophylaxis for both genders, the male participants reported they would not agree to be vaccinated. When asked about the availability of the HPV vaccine, one male student responded, "Don't worry, we won't take that drug at all." When asked if they would consider recommending female family members or sexual partners to get the vaccine, responses were typically non-committal, such as "I don't know," "It's like a personal choice," and "(Females) have the option of getting the shot if (they) want to." The majority of the male participants expressed views that females were the ones who needed to be aware of HPV and women were perceived to be exclusively responsible for HPV prevention and control options, not males.

\section{Cultural barriers}

Cultural barriers had a significant influence on sexual health education, communication, and participation in HPV education and prevention programs for the American Indian male students. In discussions about HPV and cultural barriers, it was explained that discussing HPV could be considered a taboo. Male participants explained that cultural discomfort could be expected in a discussion about HPV within most American Indian communities: "it would 
automatically lead to the big question (sexuality and pregnancy)." A male student explained the difficulties he perceived in having open communication with American Indian parents, describing how discussions about sexuality or sexual health would be heavily influenced by generational differences: "Traditionally, things like this (HPV infection) were not very prevalent in (my mother's) time. She wouldn't really know what to say... she was raised differently." Another male added, "My dad is more traditional than my mom and I would probably talk to my mom about (sexuality)." Others explained that "(My family) would just not bring it up because of religion." The students expressed the importance of upholding Native traditions, noting that American Indian beliefs and religiosity commonly prohibit open discussion about sexuality, especially across genders and among close family members.

Aside from generational differences and traditional beliefs, one participant believed that his parents had not discussed sexuality because of the expectation that the school system, not the parents, should provide sex education: "My parents never really talked to me, they understood that I learned it there (sex education in school)." Many male students reiterated a similar sentiment, emphasizing that it was preferred, as well as expected, that sex education be offered in the school system. Thus the male participants overwhelming voiced their preference for school educators to teach them regarding the HPV transmission, health consequences, risks, prevention and vaccinations over relatives or parents.

\section{Intention to modify behavior}

Information was collected about American Indian males' intention to modify their behavior by either receiving or accepting HPV education (control), and/or practicing safer sexual behaviors. Few of the participants expressed the intention to modify their behavior to incorporate prevention and control (safer sexual practices) activities. The importance of safer sexual practices was downplayed because the male students perceived they had low susceptibility to HPV infection. One male stated, "It's like you might not be affected" (so why take these measures). The male students reported use of "protection"; condoms and abstinence were identified as methods for prevention of STIs. Our study found that few of the American Indian males had intentions to modify safer sex practices and/or modify their ideas about HPV prevention, largely due to low risk perception and low perceived responsibility. Lack of readiness and maturity, coupled with distrust of the HPV vaccine and the Western healthcare systems was presented by a male student. He pointed out that, "They explain a drug to you at that age (6th grade); you're still not going to pay attention. How many at that age want to take a drug?" Several males reacted with distrust of the HPV vaccine for women, and echoed more suspicions when the possibility of HPV prophylaxis for males was discussed. The possibility of adverse reactions prompted a students to state, "I would probably like to hear (more information about vaccine) from someone that does studies and different research on that issue" (aside from companies marketing the vaccine) prior to agreeing to a vaccine. Another shared his opinion that, "It might be deadly in the future, you don't know," a point shared by several males.

\section{Discussion}

Our study findings highlighted the first exposure to information about HPV infection for many of the male participants. As a result, we observed male posturing when knowledge levels were low, low perceived responsibility, and very limited intention to adopt safer sexual risk behaviors among male American Indian college students. Their low perceptions of risk of HPV infection and a strong denial of personal responsibility regarding HPV prevention and transmission is likely due to lack of knowledge regarding the transmission, health consequences, and ways to prevent HPV infection. A study by Gerend and Barley showed that informing males of the benefits of the HPV vaccine for their female partner(s) did not increase male vaccine acceptability any more that providing them with information on the benefits to men alone [19]. Reactions from the focus groups indicate that recommendations of HPV vaccines for men may not be acceptable to many American Indian males until knowledge of HPV prevention is more broadly available and better accepted by the general public. Our study findings are similar to others who show that HPV knowledge levels are low among minority groups, as well as among males [20,21]. We found that American Indian males' low HPV knowledge did appear to influence their attitude (low risk perception) and prevention behaviors (poor acceptance of HPV vaccine for female counterparts, low perceived responsibility for safer sexual behaviors, and minimal intention to modify behaviors). Poor knowledge may have resulted in poor intention for HPV prevention and control, which could also impact high risk behaviors, such as sex with multiple partners and sex without protection, facilitating the spread of HPV and other STIs.

Recognition of how men and women accept responsibility for sexual health decisions and the re-shaping of perceptions about personal HPV/risk may be accomplished through sexual health education targeting the risks of all HPV-related cancers and genital warts. Another reason behind low perceived responsibility and low intention to modify sexual risk behaviors may include previous and continued emphasis of HPV prevention and vaccination programs for females. The unanticipated influence of gender targeting of HPV vaccination programs needs further attention to assure the protection of both genders. Future research studies can be helpful to determine if this influence is long-lasting and to measure how HPV vaccine targeting has influenced its acceptability for each gender.

Lack of information or misinformation about HPV has been identified in this study, as has the role of males in prevention activities, such as safer sexual behaviors and potential future vaccination in young men. Lenient attitudes regarding HPV prevention and transmission, and poor personal risk perceptions may have contributed to the males' negative intention to modify their sexual behaviors.

Our findings also point to the need for a better understanding by researchers and educators of contextual influences like cultural values, communication patterns and long-standing taboos among young American Indians with regard to HPV, STIs, and discussions about sexuality in general. Female vaccination may reduce infection rates among the general population, but this must be coupled with targeted, culturally-sensitive education and prevention programs for American Indian males. Clearly expanding STI and sexual health education programs serving American Indian communities is necessary to address knowledge needs and to correct misunderstandings, to change low perceptions of risk of HPV infection, and to increase perceived personal responsibility among young males and their parents. HPV prevention and control programs (vaccines, safer sexual behaviors, etc.) must incorporate cultural aspects of American Indian pedagogy, such as the role of male as protector of the family (therefore preventing spread of disease to loved ones). It is also critical to realize the restrictions inherent in cross-gender communication about STIs 
and take into account young men's need for privacy by acknowledging preferred educational delivery by school educators, rather than relatives or parents. However, the parents of young men who are ageeligible for vaccination may decide for early and timely vaccination, prior to pubertal changes of the young men.

It is likely that source of information about HPV may play an important role in shaping sexual health decision-making strategies and perceptions of responsibility; however, more research in this area is needed. A better understanding of the cultural values of American Indian communities regarding education and discussions regarding sexual behaviors (within the context of harmony of the body and spirit), prevention activities, and risks and responsibilities (to protect the health of the individual and the future of the tribe) would be valuable in developing HPV educational programs for both genders.

The federal Advisory Committee on Immunization Practices recommends the HPV vaccine for both girls and boys, from ages of 11 and 12 (and even as early as 9) to age 21 [2] We recommend schoolbased culturally sensitive HPV prevention programs that include improving responsible safer sexual practices by instructing males in activities to reduce cancer and STI disease burden among youth. Emphasis on preventing genital warts, other STIs, as well as HPVrelated cancers found in males and females may increase acceptability and responsibility of HPV prevention measures among males. Should males not receive the HPV vaccine earlier, they can catch up on the 3series vaccination in their teens and early 20 s.

This study had some limitations that could prove important opportunities for future research. First, the HPV vaccine was not licensed for males when this study was done, thus we could not adequately assess potential interventions. Now that Gardasil is licensed and is indicated for males, researchers can better assess whether factors we identified are important barriers to HPV prevention and control activities (vaccines and safer sexual behaviors). Secondly, our study population was limited to American Indian college students at four colleges/universities in the west. It will be important to extend studies to larger samples of American Indian in rural and urban populations and among several cohorts of males so that generalizability can be accomplished.

Future studies should evaluate HPV prevention and intervention studies that have equal representation of males. Males can and should participate in HPV prevention programs as males may reduce cancer and STI disease burden in both genders. Emphasis on preventing genital warts, other increasingly serious STIs, such as HIV, as well as HPV-related cancers found in males and females may increase acceptability of HPV prevention measures among males. More attention must be paid to inform the public that reducing HPV infection in males reduces the cervical cancer risks in females and reduces the risks of genital warts and cancers of the penis, anus and oropharyngeal areas among males.

\section{References}

1. CDC (2011) Recommendations on the Use of Quadrivalent Human Papillomavirus Vaccine in Males-Advisory Committee on Immunization Practices (ACIP). MMWR 60: 1705-1708.

2. http://www.gardasil.com/what-is-gardasil/index.html.

3. http://www.cdc.gov/std/hpv/stdfact-hpv-and-men.htm.

4. http://www.fda.gov/NewsEvents/Newsroom/PressAnnouncements/ ucm187003.htm.

5. Kaufman CE, Shelby L, Mosure DJ, Marrazzo J, Wong D, et al. (2007) Within the hidden epidemic: sexually transmitted diseases and HIV/AIDS among American Indians and Alaska Natives. Sex Transm Dis 34: 767-777.

6. Alfonsi GA, Datta SD, Mickiewicz T, Koutsky LA, Ghanem K, et al. (2011) Prevalence of high-risk HPV types and abnormal cervical cytology in American Indian/Alaska Native women, 2003-2005. Public Health Rep 126: 330-337.

7. Clifford GM, Gallus S, Herrero R, Munoz N, Snijders PJ, et al. (2005) Worldwide distribution of human papillomavirus types in cytologically normal women in the International Agency for Research on Cancer HPV prevalence surveys: a pooled analysis. Lancet 366: 991-998.

8. Shalala DE, Trujillo MH, Harry RH, Skupien MB, DAngelo AJ (1997) Regional Differences in Indian Health. Indian Health Service.

9. www.cdc.gov/std/hpv/common/ai/ai.pdf.

10. http://www.cancer.gov/cancertopics/factsheet/risk/HPV.

11. http://www.cdc.gov/std/hpv/stdfact-hpv-and-men.htm.

12. http://abcnews.go.com/Health/Wellness/rise-hpv-related-throat-cancersmen/story?id=13734582

13. Gonik B (2006) Strategies for fostering HPV vaccine acceptance. Infect Dis Obstet Gynecol 2006 Suppl: 36797.

14. McPartland TS, Weaver BA, Lee SK, Koutsky LA (2005) Men's perceptions and knowledge of human papillomavirus (HPV) infection and cervical cancer. J Am Coll Health 53: 225-230.

15. Yacobi E, Tennant C, Ferrante J, Pal N, Roetzheim R (1999) University students' knowledge and awareness of HPV. Prev Med 28: 535-541.

16. Zimet GD, Mays RM, Winston Y, Kee R, Dickes J, et al. (2000) Acceptability of human papillomavirus immunization. J Womens Health Gend Based Med 9: 47-50.

17. Glaser BG, Strauss AL (1967) The Discovery of Grounded Theory: Strategies for Qualitative Research. Chicago, Aldine Publishing Company.

18. Gerend MA, Barley J (2009) Human papillomavirus vaccine acceptability among young adult men. Sex Transm Dis 36: 58-62.

19. Klug SJ, Hukelmann M, Blettner M (2008) Knowledge about infection with human papillomavirus: a systematic review. Prev Med 46: 87-98.

20. Reiter P, Brewer NT, Smith JS (2010) HPV Knowledge and HPV Vaccine Acceptability among a National Sample of Heterosexual Males. Sex Transm Infect 86: 241-246.

21. McRee AL, Reiter PL, Chantala K, Brewer NT (2010) Does framing human papillomavirus vaccine as preventing cancer in men increase vaccine acceptability? Cancer Epidemiol Biomarkers Prev 19: 1937-1944. 